\title{
Central Trochlear Nerve Palsy due to Stroke: Report and Clinical Correlation of Two Cases
}

\author{
Luis A. Robles
}

Keywords: brain injury, carotid artery disease, cerebral trauma, cerebrovascular disease

Head injury is the principal acquired cause of trochlear nerve (TN) palsy; other causes of TN palsy include surgical injury, inflammatory processes, tumors and stroke. Reports of isolated trochlear palsy due to mesencephalic stroke are extremely uncommon. ${ }^{1-4}$ This paper presents two different cases of central TN palsy caused by stroke in the midbrain, which manifested in different ways.

\section{Case Reports}

\section{Case 1}

An elderly lady, 80 years of age, with a past medical history of high blood pressure was admitted to the emergency department after sudden onset of dizziness and binocular diplopia. Dizziness improved in the next 24 hours but diplopia did not change. She was discharged from the hospital with medical treatment. She was initially checked two months later because of persistence of binocular diplopia. Diplopia was vertical when she focused downwards. She did not present any other neurological symptom.

On examination, mild left hypertropia was observed in primary gaze, which increased with left head tilt (positive Bielschowsky's test); the hypertropia was more evident in the right down gaze. According to the patient, diplopia was vertical and it was maximal in the right down gaze. Examination on the rest of the oculomotor nerves was normal. Pupils were equal, normally reacting to light. No other cranial nerves were affected. There were no motor, cerebellar or sensory abnormalities in the limbs.

All clinical findings were compatible with left isolated trochlear nerve palsy.

Brain MRI revealed a T2-weighted hyper intensity within the right dorsal midbrain at the level of colliculus inferior, suggestive of an ischemic event. A schematic drawing representing the stroke location is also shown (Figure 1). Magnetic resonance imaging angiogram was normal. Treatment was initiated with Clopidogrel, and prisms to improve diplopia were prescribed by the ophthalmologist. The diplopia completely resolved over the following six months.

\section{Case 2}

This is a 61 years previously healthy man. Symptoms started while he was exercising at the gym. He suddenly experienced a right frontal headache, accompanied by dizziness and double vision. He was initially checked a week later because of persistence of double vision especially when looking downwards and to the left; in addition, he complained of numbness of left arm and left side of face. The patient also noticed that his right eye was smaller than the left eye and he mentioned having had several episodes of tinnitus from the onset of this condition.

On examination, the head position was normal. Ophthalmologic assessment revealed mild right ptosis and miosis; full-appearing ocular ductions, left mild hypertropia was observed in primary gaze and increased during right down gaze. Diplopia was more important on right down gaze and improved when head was tilted on to the right side. Hearing was clinically normal. Pain and temperature sensation were decreased in the left arm and left side of face. No cerebellar nor motor abnormalities in the limbs were found.

Brain magnetic resonance imaging showed a T1 hyperintense lesion located in the right dorsal midbrain, suggestive of a hemorrhagic stroke. A schematic drawing representing the stroke location is also depicted (Figure 2).

All clinical findings were compatible with left trochlear nerve palsy, right Horner's syndrome, right trigeminal pathway lesion, right anterolateral system lesion and probably dysfunction in the auditory pathways. Prisms were provided by the ophthalmologist to improve diplopia.

\section{Discussion}

Figures 1 and 2 show the anatomic position of the trochlear nerve nucleus. The trochlear nucleus is located in the tegmentum of the caudal midbrain. It lies inferior to the oculomotor nucleus and ventro-lateral to the aqueduct, at the level of the inferior colliculus. The trochlear nerve fascicle courses around the aqueduct to decussate in the superior medullar velum of the dorsal midbrain and exits in the contralateral side below the inferior colliculus. The $\mathrm{TN}$ is the only cranial nerve whose fibers cross over as they emerge from the midbrain; therefore, clinical manifestations of disturbance of this nerve are contralateral when the affection is localized in the midbrain. The medial longitudinal fasciculus (MLF) is located immediately anterior to the TN nucleus; for this reason, when the TN nucleus is affected, patients frequently show an associated internuclear ophtalmoplegia secondary to involvement of this tract. Since the trochlear nucleus and fascicle are surrounded by other tracts, clinical presentation may vary

From the Section of Neurology and Neurosurgery, Hospital Amerimed, Blvb Fco Medina Ascencio 3970, Puerto Vallarta, Mexico, CP 48335.

Received May 1, 2015. Final Revisions Submitted October 5, 2015.

Correspondence to: Luis A. Robles, Section of Neurology and Neurosurgery, Hospital

Amerimed, Blvb Fco Medina Ascencio 3970, Puerto Vallarta, Mexico, CP 48335.

Email: larob@prodigy.net.mx 

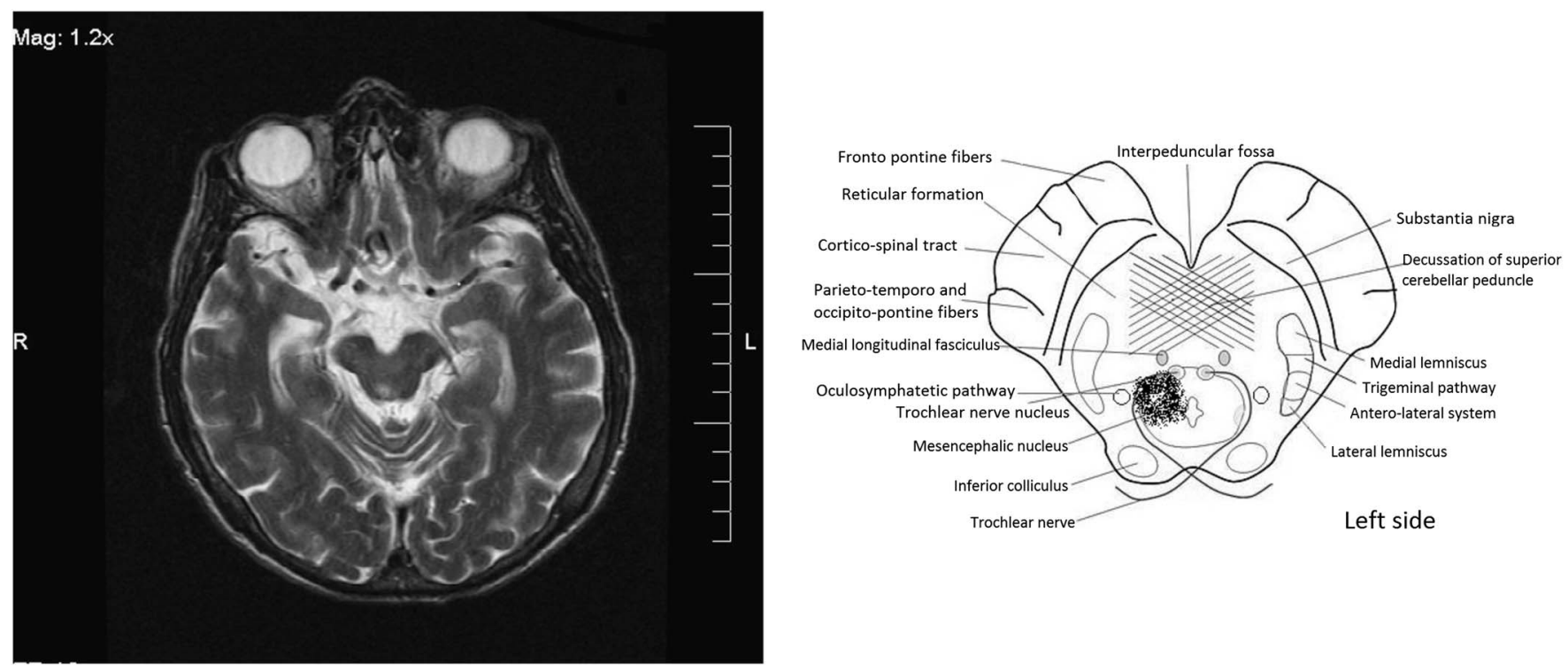

Figure 1: Left; axial brain MRI (T2 sequence) at the level of inferior colliculus. It shows a right small hyperintense ischemic lesion located lateral and slightly anterior to the aqueduct. Right; schematic drawing representing the lesion. The stroke area looks splattered in the image. Note that the stroke is located in the area where part of TN nucleus and fascicle are located.

depending on which pathways are affected. Several ascendant sensorial tracts are located in the lateral border of tegmental zone, from posterior to anterior they are: lateral lemniscus, anterolateral tract, trigeminal pathway (trigeminothalamic pathway) and the medial lemniscus. The oculosympathetic tract descending from the hypothalamus is located between the $\mathrm{TN}$ nucleus and the already mentioned ascendant sensorial pathways.

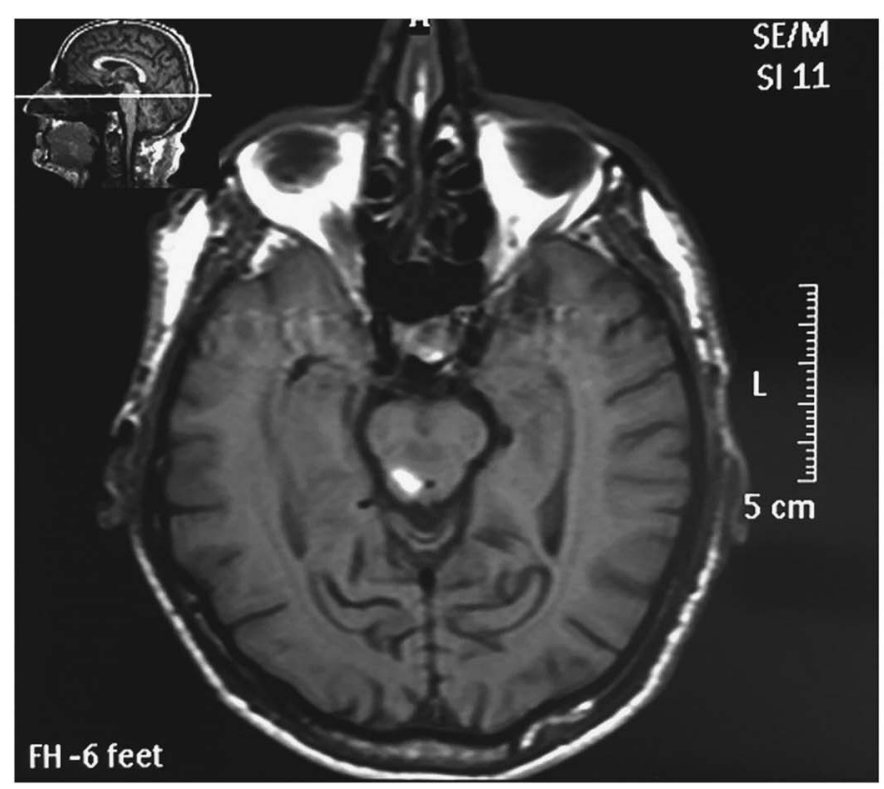

In Case 1 , the patient showed an isolated left central TN palsy secondary to a small ischemic stroke in the right dorsal midbrain. The lesion was located lateral and slightly anterior to the aqueduct, very likely affecting part of the TN nucleus and fascicle (Figure 1). Because the size of the lesion was very small and was located in this very specific area, it did not affect other adjacent midbrain tracts.

Figure 2: Left; axial brain MRI (T1 sequence) at the level of inferior colliculus. It shows a right hyperintense lesion compatible with hemorrhage. The lesion is located lateral to the aqueduct and extends to the lateral border of the midbrain. Right; schematic drawing showing the lesion and affected structures. The stroke area looks splattered in the image. The hemorrhage is affecting the TN fascicle, oculosympathetic tract, lateral lemniscus, trigeminal and anterolateral pathways. Note that the TN nucleus, medial longitudinal fasciculus and medial lemniscus are not compromised. 
In Case 2, the patient experienced a right midbrain hemorrhage manifested by a left central TN palsy and a variety of neurological signs. Figure 2 shows the location of hemorrhagic lesion. The bleeding localization was lateral to the aqueduct, and therefore, the TN nucleus and MLF were not affected. In this case, the TN palsy was secondary to an injury of the fascicle. The hemorrhage extended laterally over the tegmentum affecting several pathways. The right ptosis and miosis were secondary to an ipsilateral central Horner's syndrome caused by disturbance of the oculosympathetic tract. The presence of left hypoesthesia to pain and temperature in the face and arm were indicative of dysfunction of trigeminal and anterolateral pathways; on the other hand, the proprioceptive sensation was preserved in the arm which indicates that the medial lemniscus was not affected. Tinnitus was very likely caused by dysfunction of the lateral lemniscus.

In summary, the present paper reports two uncommon cases of central TN palsy caused by different types of stroke, showing different symptoms. A detailed pathologic-anatomical description was conducted to describe the clinical data observed in each patient.

\section{Disclosure}

Luis Robles does not have anything to disclose.

\section{REFERENCES}

1. Lee SH, Park SW, Kim BC, Kim MK, Cho KH, Kim JS. Isolated trochlear palsy due to midbrain stroke. Clin Neurol Neurosurg. 2010;112:68-71.

2. Hatori K, Urabe T, Kanazawa A, Mizuno Y. A case of brainstem vascular malformation with isolated trochlear nerve palsy as the initial symptom. No To Shinkei. 1991;43: 965-8.

3. Mon Y. Midbrain hemorrhage presenting with trochlear nerve palsy — a case report. Rinsho Shinkeigaku. 1991;36:71-3.

4. Thomke F, Ringel K. Isolated superior oblique palsies with brainstem lesions. Neurology. 1999;53:1126-7. 